\title{
SYNTHESIS AND ANTITUMOR ACTIVITY OF ANTHRACYCLINE DISACCHARIDE GLYCOSIDES CONTAINING DAUNOSAMINE
}

\author{
Derek Horton $^{a}$, Waldemar Priebe ${ }^{\text {b }}$, Marcos L. Sznaidman ${ }^{c}$ \\ and OSCAR VARELA ${ }^{d}$ \\ Department of Chemistry, The Ohio State University, \\ Columbus, Ohio 43210, U.S.A.
}

(Received for publication July 26, 1993)

\begin{abstract}
Daunosamine, as its 4-O-acetyl-3- $N$-triffuoroacetyl glycosyl chloride derivative (1b), has been coupled $\alpha$-L-glycosidically to the 3- and 4-mono- $O$-acetyl derivatives of L-rhamnal to afford disaccharide glycal derivatives, whose conversion into the corresponding 2-deoxyglycosides by sequential alkoxyiodination-tributylstannane reduction has been evaluated. The sequence successfully demonstrated with the methyl glycosides was successfully extended with daunomycinone as the aglycon, providing a preparative route to 7-O-[3-O-(3-amino-2,3,6-trideoxy- $\alpha-\mathrm{L}-l y \times 0$ hexopyranosyl)-2,6-dideoxy- $\alpha$-L-arabino-hexopyranosyl]daunomycinone hydrochloride (15), an analogue of natural anthracycline antibiotics containing daunosamine and a 2,6-dideoxy-L-hexose.
\end{abstract}

Most of the natural anthracycline antibiotics with oligosaccharide side chains, as in the rhodomycin and cinerubin series, contain daunosamine as the sugar directly attached to the anthracyclinone and are of interest as antitumor agents ${ }^{1)}$. Only in a few cases, daunosamine appears as the non reducing terminal sugar ${ }^{2,3)}$. These compounds also showed high degree of antitumor activity. As part of our program to obtain new anthracycline antibiotics, we decided to synthesize derivatives having daunosamine as the terminal non reducing sugar, in order to compare their biological activity with the natural products, and to be able to make conclusions about the importance of the sequence of sugars in the oligosaccharide side chain.

Synthetic analogs of daunorubicin and doxorubicin have been extensively investigated in our laboratory ${ }^{4,5}$. Conventional Koenigs-Knorr coupling between daunomycinone or 14-protected adriamycinone and appropriate glycosyl halides is sometimes complicated by low yields and mixtures of anomers. An alternative two-step synthesis ${ }^{6)}$ offers significant advantage. The first step involves trans-alkoxyhalogenation of appropriate glycal precursors with $N$-iodosuccinimide (NIS) and daunomycinone (DNM). This glycosidation method has proved useful with sugar aglycons, as demonstrated by THIEM et al. $^{7 \sim 9)}$, with DNM as shown in earlier work from our group ${ }^{10)}$, and more recently by DANISHEFSKY et al. ${ }^{11}$. The second step employs dehalogenation with tributylstannane $\left(\mathrm{Bu}_{3} \mathrm{SnH}\right)$. This general sequence was used by UMEZAWA et al. ${ }^{12)}$ to obtain simple 2-deoxy- $\alpha$-glycosides, and the steric course of dehalogenation of methyl 2-haloglycosides by $\mathrm{Bu}_{3} \mathrm{SnD}$ has been studied in detail in our laboratory ${ }^{13}$.

a Present address: Department of Chemistry, The American University, 4400 Massachusetts Avenue NW, Washington, DC 20016, U.S.A.

b Present address: The University of Texas System Cancer Center, M. D. Anderson Hospital and Tumor Institute, 1515 Halcombe Blvd., Houston, TX 77030, U.S.A.

c Present address: Department of Organic Chemistry, Burroughs Wellcome Co., Inc., 3030 Cornwallis Road, Research Triangle Park, NC 27709, U.S.A.

d Present address: Universidad de Buenos Aires, Facultad de Ciencias Exactas y Naturales, Departamento de Quimica Organica, Buenos Aires, Argentina. 


\title{
Results and Discussion
}

\author{
Chemical Synthesis
}

4-O-Acetyl-1,5-anhydro-2,3,6-trideoxy-3-trifluoroacetamido-L-lyxo-hex-1-enitol (1a) (Scheme 1), a stable, activated daunosamine derivative ${ }^{14)}$ was converted into the glycosyl chloride derivative $\mathbf{1 b}$, which was immediately coupled with 3-O-acetyl-L-rhamnal (2, readily prepared ${ }^{15)}$ by selective acetylation of L-rhamnal) under conventional typical Koenigs-Knorr conditions to afford the $\alpha$-L- $(1 \rightarrow 4)$-linked disaccharide glycal derivative 3 in $40 \%$ yield. Treatment of this glycal with hydrogen chloride in benzene, followed by Koenigs-Knorr reaction with methanol afforded only the monosaccharide methyl $\beta$-L-glycoside 4, obtained in low yield. No traces of disaccharide glycoside 7 were detected. Similar results were observed when the daunosamine derivative $1 \mathrm{~b}$ was coupled to 4 - $O$-acetyl-L-rhamnal ${ }^{15)}(\mathbf{8})$ (Scheme 2) to afford the $\alpha$-L-(1 $\rightarrow 3)$-linked disaccharide glycal derivative 9; again treatment with $\mathrm{HCl}$ and then with methanol gave only the monosaccharide glycoside 4. These results indicate that the glycal disaccharides $\mathbf{3}$ and $\mathbf{9}$ undergo hydrolysis by dry $\mathrm{HCl}$, affording the glycosyl chloride (1b) of daunosamine, which then reacts with methanol to give the glycoside 4 .

The failure of this conventional glycosidation sequence with the disaccharide glycals 3 and 9 prompted evaluation of the NIS- ROH glycosidation ${ }^{7 \sim 9)}$ and $\mathrm{Bu}_{3} \mathrm{SnH}$ dehalogenation ${ }^{12)}$ as a route to the model methyl glycosides $\mathbf{7}$ and $\mathbf{1 2}$.

Treatment of the $(1 \rightarrow 4)$-linked glycal 3 (Scheme 1) with NIS and methanol gave a 3.8:1 mixture of trans methyl 2-iodo disaccharides 5 and 6. The trans-diaxial isomer 5 was expected to be the major product, based on our previous studies concerning the stereochemistry of NIS-MeOH addition to the double bond of glycals ${ }^{16,17}$. This mixture was resolved by column chromatography and the major 1,2-trans-diaxial product (5) was deiodinated with $\mathrm{Bu}_{3} \mathrm{SnH}$ to afford the final methyl glycoside disaccharide 7 , crystalline in $73 \%$ yield.

Similarly, treatment of the $(1 \rightarrow 3$ )-linked glycal 9 (Scheme 2) with NIS-MeOH afforded a mixture of 1,2-trans isomers $\mathbf{1 0}$ and $\mathbf{1 1}$, here in 3.2:1 ratio. The major, trans-diaxial product $\mathbf{1 0}$ was likewise treated with $\mathrm{Bu}_{3} \mathrm{SnH}$ to give the methyl glycoside disaccharide 12 , crystalline in $76 \%$ yield.

Structural Assignments for Disaccharide Methyl Glycosides Derivatives 5, 6, 7, 10, 11 and 12

The products obtained by methoxyiodination of the double bond of 3 (products 5 and 6 ) and 9 (products 10 and 11), showed large $J_{3,4}(8.5 \sim 9.3 \mathrm{~Hz})$ and $J_{4,5}(8.9 \sim 9.0 \mathrm{~Hz})$ values, indicating the trans-diaxial disposition between $\mathrm{H}-3-\mathrm{H}-4$ and $\mathrm{H}-4-\mathrm{H}-5$ in the favored ${ }^{1} C_{4}(\mathrm{~L})$ conformation. The small values of $J_{2,3}(4.2 \mathrm{~Hz})$ in compounds 5 and 10 indicate the L-manno configuration. Large values of $J_{2,3}$ $(11.2 \sim 10.5 \mathrm{~Hz})$ observed for compounds 6 and 11 signify the L-gluco configuration. The large values of $J_{1,2}(8.9 \sim 9.0 \mathrm{~Hz})$ for compounds 6 and 11 likewise accord with their having the $\beta$-L-gluco configuration. Small $(1.4 \sim 0 \mathrm{~Hz}) J_{1,2}$ coupling constants, again support assignment of the $\alpha$-L-manno configuration to compounds 5 and 10. The structural assignment for compounds 5 and $\mathbf{1 0}$ was further consolidated from $\mathrm{NMR}$ data on their $\mathrm{Bu}_{3} \mathrm{SnH}$ reduction-products, compounds 7 and 12, respectively. Both of these 2-deoxyglycosides showed two small coupling constants between $\mathrm{H}-1$ and the protons at C-2 $\left(J_{1,2 \text { eq }}\right.$ close to zero and $J_{1,2 \mathrm{ax}}=3.7,3.1 \mathrm{~Hz}$ for 7 and 12 , respectively), indicating that $\mathrm{H}-1$ is equatorial. Compounds 7 and 12 also showed large $J_{3,4}(9.1 \sim 9.3 \mathrm{~Hz})$ and $J_{4,5}(9.1 \sim 9.5 \mathrm{~Hz})$ values, indicating the trans-diaxial disposition between $\mathrm{H}-3-\mathrm{H}-4$ and $\mathrm{H}-4-\mathrm{H}-5$ in the favored ${ }^{1} C_{4}(\mathrm{~L})$ conformation. 
Scheme 1
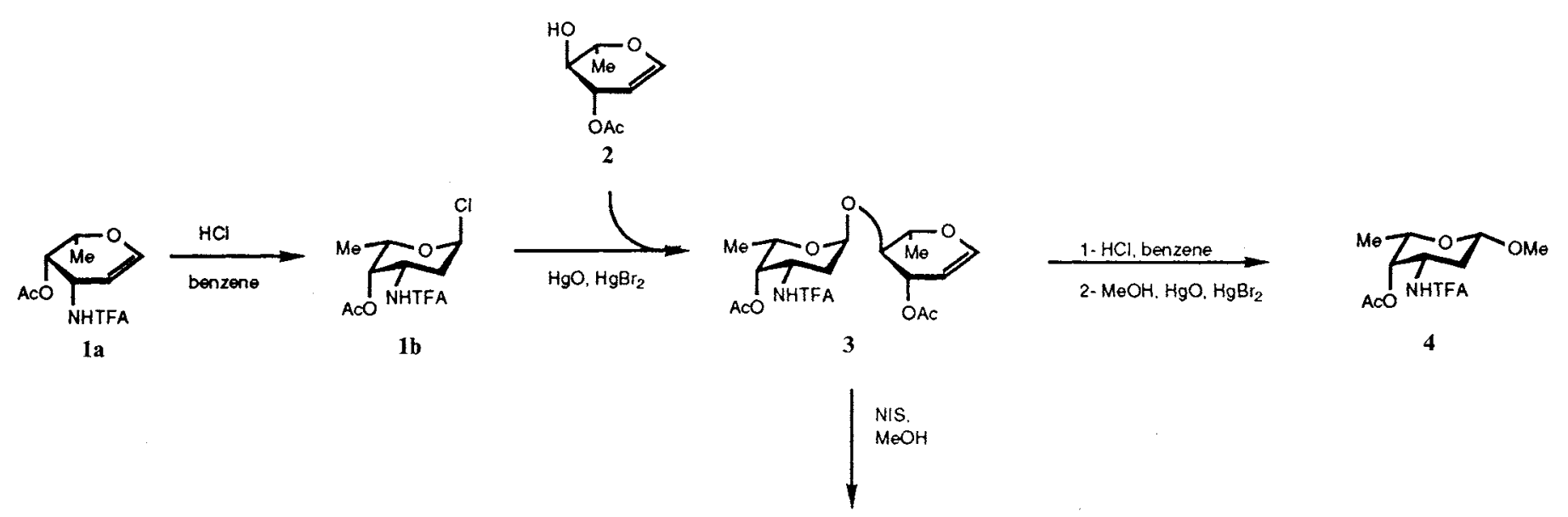

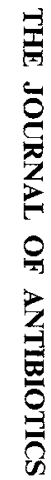

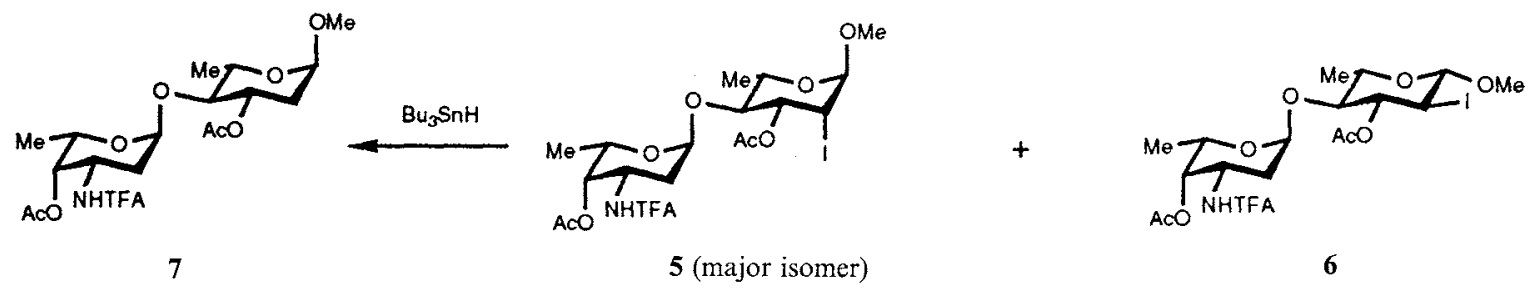


Scheme 2.
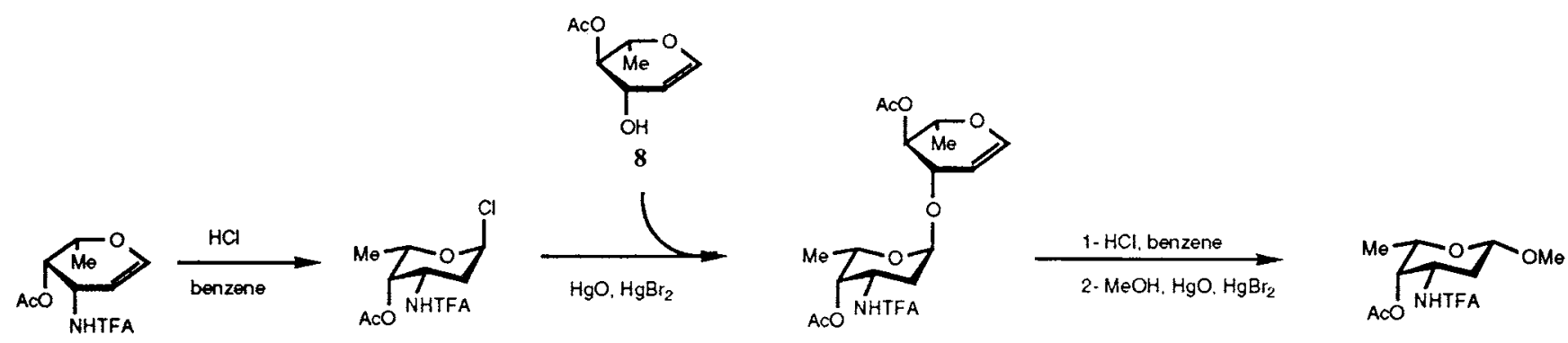

1b

9

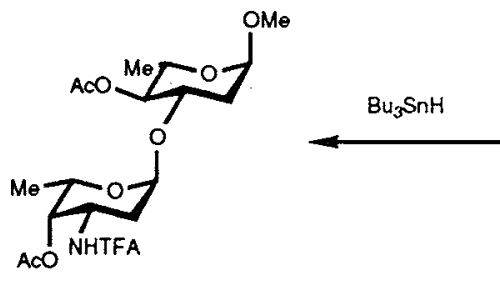

12

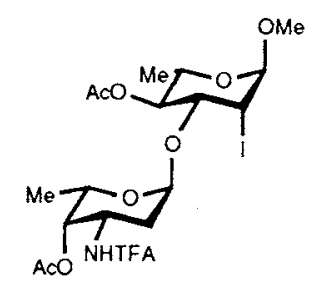

10 (major isomer)

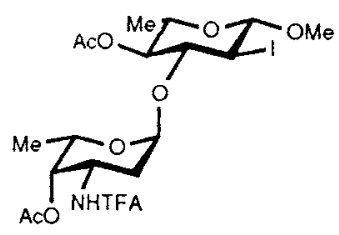

11

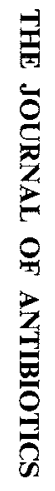

NIS: $N$-iodosuccinimide 


\section{Glycosidation of 9 with Daunomycinone}

Alkoxyiodination of disaccharide glycal 9 (Scheme 3) with NIS-DNM afforded the trans-diaxial addition product 13 as the only observed product, isolated crystalline in $50 \%$ yield. Deiodination by $\mathrm{Bu}_{3} \mathrm{SnH}$ gave the 2-deoxyglycoside 14 in $55 \%$ yield. Deprotection of 14 by transesterification with NaOMe and then amide hydrolysis with aqueous $\mathrm{NaOH}$, afforded, after crystallization from $\mathrm{HCl}-\mathrm{MeOH}$ and ether, the target anthracycline disaccharide glycoside $\mathbf{1 5}$ in $32 \%$ yield as a crystalline product.

\section{Structural Assignments for Compounds 13, 14 and 15}

The alkoxyiodination product 13 derived from disaccharide glycal 9 showed large $J_{3^{\prime}, 4^{\prime}}(9.3 \mathrm{~Hz})$ and $J_{4^{\prime}, 5^{\prime}}(9.3 \mathrm{~Hz})$ couplings, indicating the trans-diaxial disposition of $\mathrm{H}-3^{\prime}-\mathrm{H}^{\prime} 4^{\prime}$ and $\mathrm{H}-4^{\prime}-\mathrm{H}-5^{\prime}$ in the favored ${ }^{1} C_{4}$ (L) conformation. The small value of $J_{2,3^{\prime}}(4.0 \mathrm{~Hz})$, indicates the L-manno configuration, and the small $J_{1^{\prime}, 2^{\prime}}(1.4 \mathrm{~Hz})$ coupling constant indicates the $\alpha$-L-anomeric configuration. The last assignment was confirmed by studies on the deiodination product 14. Compound 14 showed two small coupling constants between $\mathrm{H}-1^{\prime}$ and the hydrogen atoms at $\mathrm{C}-2^{\prime}\left(J_{1^{\prime}, 2^{\prime} \mathrm{eq}}=\sim 0\right.$ and $\left.J_{1^{\prime}, 2^{\prime} \mathrm{ax}}=3.4 \mathrm{~Hz}\right)$, indicating that $\mathrm{H}-1^{\prime}$ is equatorial. Compound 14 also showed large $J_{3^{\prime}, 4^{\prime}}(9.1 \mathrm{~Hz})$ and $J_{4^{\prime}, 5^{\prime}}(9.4 \mathrm{~Hz})$ values, further supporting the trans-diaxial disposition between $\mathrm{H}-3^{\prime}-\mathrm{H}^{-} 4^{\prime}$ and $\mathrm{H}-4^{\prime}-\mathrm{H}-5^{\prime}$ in the favored ${ }^{1} \mathrm{C}_{4}$ (L) conformation. The final deprotected product, compound 15, shows the same coupling pattern as 14: small coupling constants between $\mathrm{H}-1^{\prime}$ and $\mathrm{C}-2^{\prime}$ protons $\left(J_{1^{\prime}, 2^{\prime} \text { eq }}=\sim 0\right.$ and $\left.J_{1^{\prime}, 2^{\prime} \text { ax }}=3.3 \mathrm{~Hz}\right)$, confirming the equatorial disposition of $\mathrm{H}-1^{\prime}$. The large couplings between $\mathrm{H}-3^{\prime}-\mathrm{H}-4^{\prime}\left(J_{3^{\prime}, 4^{\prime}}=9.1 \mathrm{~Hz}\right)$ and $\mathrm{H}-4^{\prime}-\mathrm{H}-5^{\prime}\left(J_{4^{\prime}, 5^{\prime}}=8.5 \mathrm{~Hz}\right)$, confirm the trans-diaxial disposition of these hydrogens in the favored ${ }^{1} C_{4}(\mathrm{~L})$ conformation.

\section{Biological Testing}

The disaccharide glycoside 15 (NSC 6549947) was submitted to the National Cancer Institute for testing in the human cell line-based in vitro anticancer screen, and was tested on two separate occasions. The tests involved exposure to close to 60 cell lines. The compound showed no appreciable cell-line

Scheme 3.
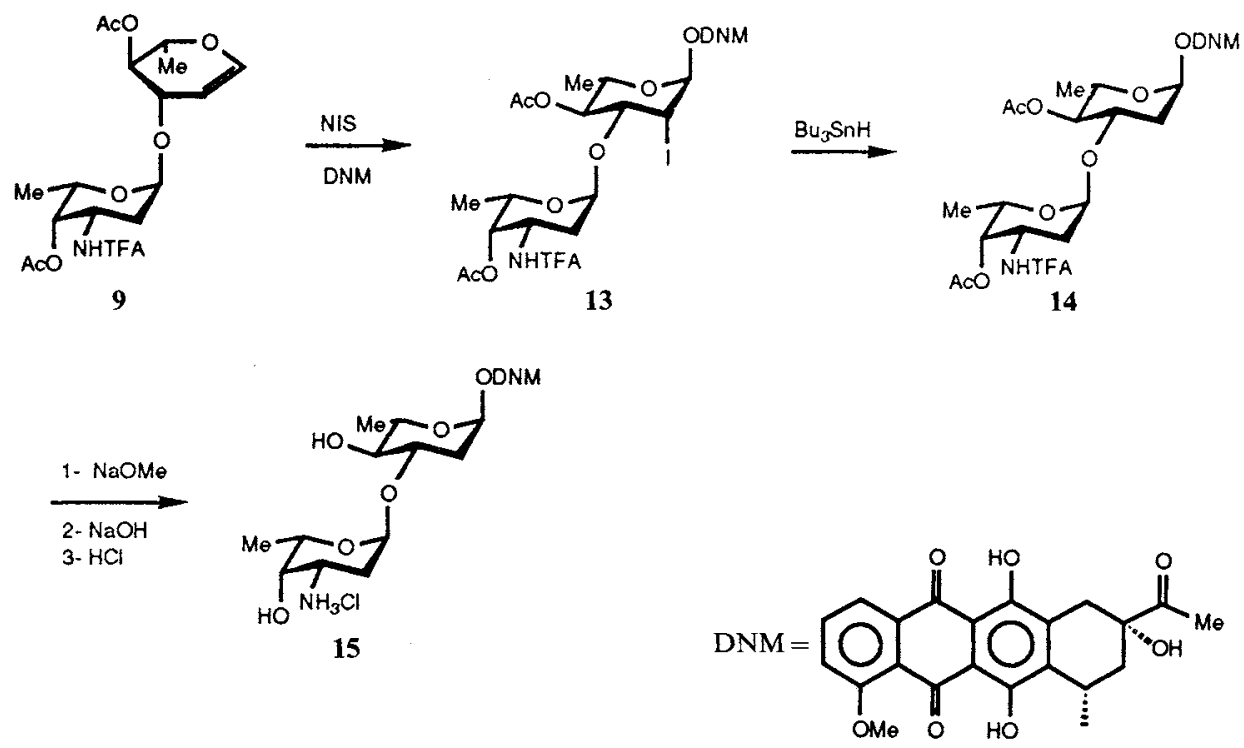

NIS: $\quad N$-iodosuccinimide

DNM: daunomycinone 
selectivity, with total growth inhibition (- $\log [$ concentration] $)$ against all lines of $>4$. The $\mathrm{IC}_{50}$ value against all lines was 4.01 . The compound thus displayed only marginal activity.

\section{Conclusions}

This work demonstrates a potentially general and effective synthetic method for producing an oligosaccharide terminated at the reducing end by a glycal, and coupling this in a two-step sequence to a sensitive, highly functionalized alcohol, to produce the corresponding 2-deoxyglycoside of the alcohol and the oligosaccharide. The approach is shown to be particularly effective in synthesis of anthracycline glycosides.

\section{Experimental}

\section{General Methods}

Solvents were dried and redistilled just prior to use. Melting points were determined in open glass capillaries by using a Thomas-Hoover apparatus, and are uncorrected. Optical rotations were measured with a Perkin-Elmer Model 141 polarimeter, ${ }^{1} \mathrm{H}$ NMR spectra were recorded at $500 \mathrm{MHz}$ with a Bruker AM-500 spectrometer by C. E. CoTtrell. The samples were dissolved in the indicated solvent and the chemical shifts $(\mathrm{ppm})$ refer to an internal standard of tetramethylsilane $(\delta=0.0 \mathrm{ppm})$. All signal assignments for the disaccharide glycals and methyl glycosides were verified by ${ }^{13} \mathrm{C}-{ }^{1} \mathrm{H}$ correlation experiments. Evaporations were performed under vacuum. TLC was performed on precoated aluminum sheets $(0.2 \mathrm{~mm})$ and glass plates $\left(0.25 \mathrm{~mm}\right.$ ) coated with Silica gel $60 \mathrm{~F}_{254}$ (E. Merck, Darmstadt); components were detected by spraying the plates with $0.1 \mathrm{M}$ ceric sulfate in $2 \mathrm{M}$ sulfuric acid, with subsequent heating. Column chromatography was performed with Silica gel 60 (230 400 mesh, E. Merck, Darmstadt). High resolution FAB mass spectra were recorded in a Kratos MS-30 apparatus by Mr. C. R. WeISENBERGER. Elemental analysis were determined by Atlantic Microlab (Atlanta, GA).

4-O-(4-O-Acetyl-2,3,6-trideoxy-3-trifluoroacetamido- $\alpha$-L-lyxo-hexopyranosyl)-3-O-acetyl-1,5-anhydro-2,6-dideoxy-L-arabino-hex-1-enitol (3)

4-O-Acetyl-1,5-anhydro-2,6-dideoxy-3-trifluoroacetamido-L-lyxo-hex-1-enitol ${ }^{14)}$ (1a) $(1.0 \mathrm{~g}, 3.7 \mathrm{mmol})$ was dissolved in dry benzene $(100 \mathrm{ml})$ and the solution evaporated to half its volume. $\mathrm{HCl}$ gas was then bubbled through the magnetically stirred solution for 10 minutes at $5^{\circ} \mathrm{C}$. After 5 minutes at room temperature, the solvent was evaporated. The residue was redissolved in dry $\mathrm{CH}_{2} \mathrm{Cl}_{2}$, the solution evaporated, and the residue redissolved again, affording a solution of the glycosyl chloride 1 b in dry $\mathrm{CH}_{2} \mathrm{Cl}_{2}$.

At the same time, $\mathrm{HgO}(2.31 \mathrm{~g}, 10.7 \mathrm{mmol}), \mathrm{HgBr}_{2}(1.48 \mathrm{~g}, 4.1 \mathrm{mmol})$ and powdered $4 \AA$ molecular sieves $(3.63 \mathrm{~g})$ were mixed in dry $\mathrm{CH}_{2} \mathrm{Cl}_{2}(70 \mathrm{ml})$ and stirred magnetically for 1 hour at room temperature. To this mixture were succesively added a solution of $3-O$-acetyl-L-rhamnal ${ }^{15)}(2,1.32 \mathrm{~g}, 7.6 \mathrm{mmol})$ in dry $\mathrm{CH}_{2} \mathrm{Cl}_{2}(10 \mathrm{ml})$ and then the solution of glycosyl chloride $\mathbf{1 b}$. The mixture was magnetically stirred at room temperature with TLC monitoring (2:1 hexane-EtOAc). After 2 hours the mixture was filtered through Celite and the filtrate was successively washed with $30 \%$ aqueous $\mathrm{KI}$, twice with water, and then dried $\left(\mathrm{Na}_{2} \mathrm{SO}_{4}\right)$ and evaporated. The resultant syrup was resolved by column chromatography $(5: 1$ hexane-EtOAc) to afford the disaccharide derivative 3 as a solid $(640 \mathrm{mg}, 39 \%)$. Compound 3 was crystallized from $\mathrm{Et}_{2} \mathrm{O}$ - hexane; $\mathrm{mp} 144 \sim 146^{\circ} \mathrm{C} ;[\alpha]_{\mathrm{D}}^{20}-80.0^{\circ}\left(c 1.0, \mathrm{CHCl}_{3}\right) ;{ }^{1} \mathrm{H} \mathrm{NMR}\left(\mathrm{CDCl}_{3}\right): \delta 6.40$ (dd, $1 \mathrm{H}, J_{1.2}=6.1$ and $\left.J_{1,3}=1.0 \mathrm{~Hz}, \mathrm{H}-1\right), 6.23\left(\mathrm{~d}, 1 \mathrm{H}, J_{3^{\prime}, \mathrm{NH}}=7.2 \mathrm{~Hz}, \mathrm{~N}-\mathrm{H}\right), 5.12 \sim 5.26(\mathrm{~m}, 3 \mathrm{H}, \mathrm{H}-3$, $\mathrm{H}-1^{\prime}$ and $\left.\mathrm{H}-4^{\prime}\right), 4.75\left(\mathrm{dd}, 1 \mathrm{H}, J_{2,3}=3.3 \mathrm{~Hz}, \mathrm{H}-2\right), 4.51\left(1 \mathrm{H}, \mathrm{m}, \mathrm{H}-3^{\prime}\right), 4.15\left(\mathrm{q}, 1 \mathrm{H}, J_{5^{\prime}, 6^{\prime}}=6.5 \mathrm{~Hz}, \mathrm{H}-5^{\prime}\right)$, $4.10\left(\mathrm{~m}, 1 \mathrm{H}, J_{5,6}=6.7 \mathrm{~Hz}, \mathrm{H}-5\right), 3.78\left(\mathrm{dd}, 1 \mathrm{H}, J_{3,4}=5.4\right.$ and $\left.J_{4,5}=7.1 \mathrm{~Hz}, \mathrm{H}-4\right), 2.17\left(\mathrm{~s}, 3 \mathrm{H}, \mathrm{CH}_{3} \mathrm{CO}\right)$, 2.06 (s, 3H, $\left.\mathrm{CH}_{3} \mathrm{CO}\right), 1.91 \sim 1.94\left(\mathrm{~m}, 2 \mathrm{H}, \mathrm{H}-2^{\prime} \mathrm{ax}\right.$ and $\left.\mathrm{H}-2^{\prime} \mathrm{eq}\right), 1.38\left(\mathrm{~d}, 3 \mathrm{H}, \mathrm{CH}_{3}\right), 1.13\left(\mathrm{~d}, 3 \mathrm{H}, \mathrm{CH}_{3}{ }^{\prime}\right)$; ${ }^{13} \mathrm{C} \mathrm{NMR}\left(\mathrm{CDCl}_{3}\right): \delta 170.8\left(\mathrm{CH}_{3} \mathrm{CO}\right), 170.4\left(\mathrm{CH}_{3} \mathrm{CO}\right), 145.9(\mathrm{C}-1), 98.4(\mathrm{C}-2), 96.4\left(\mathrm{C}-1^{\prime}\right), 75.7(\mathrm{C}-4)$, $73.2(\mathrm{C}-5), 70.2$ (two overlapped signals $\mathrm{C}-3$ and $\left.\mathrm{C}^{\prime} 4^{\prime}\right), 65.8\left(\mathrm{C}-5^{\prime}\right), 45.1\left(\mathrm{C}-3^{\prime}\right), 30.2\left(\mathrm{C}-2^{\prime}\right), 21.1\left(\mathrm{CH}_{3} \mathrm{CO}\right)$, $20.5\left(\mathrm{CH}_{3} \mathrm{CO}\right), 17.5(\mathrm{C}-6), 17.1\left(\mathrm{C}-6^{\prime}\right)$.

Anal Calcd for $\mathrm{C}_{18} \mathrm{H}_{24} \mathrm{~F}_{3} \mathrm{NO}_{8}: \quad \mathrm{C} 49.20, \mathrm{H} 5.51, \mathrm{~N} 3.19$. Found: 
4-O-Acetyl-3-O-(4-O-acetyl-2,3,6-trideoxy-3-trifluoroacetamido- $\alpha$-L-lyxo-hexopyranosyl)-1,5-anhydro-2,6-dideoxy-L-arabino-hex-1-enitol (9)

The same procedure was used as for compound 3, except that 4-O-acetyl-L-rhamnal ${ }^{15)}(\mathbf{8})$ was used instead of its 3-O-acetyl isomer 2. A solid was obtained which was crystallized from $\mathrm{Et}_{2} \mathrm{O}$-hexane to afford pure $9(820 \mathrm{mg}, 50 \%) ; \mathrm{mp} 184 \sim 185^{\circ} \mathrm{C} ;[\alpha]_{\mathrm{D}}^{20}-73^{\circ}\left(c 0.44, \mathrm{CHCl}_{3}\right) ;{ }^{1} \mathrm{H}$ NMR $\left(\mathrm{CDCl}_{3}\right): \delta 6.41(\mathrm{dd}$, $1 \mathrm{H}, J_{1,2}=6.2$ and $\left.J_{1,3}=1.3 \mathrm{~Hz}, \mathrm{H}-1\right), 6.34(\mathrm{br} d, 1 \mathrm{H}, \mathrm{N}-\mathrm{H}), 5.16\left(\mathrm{~m}, 1 \mathrm{H}, \mathrm{H}-1^{\prime}\right), 5.12\left(\mathrm{~m}, 1 \mathrm{H}, \mathrm{H}-4^{\prime}\right), 4.97$ (dd, $1 \mathrm{H}, J_{3,4}=4.6$ and $\left.J_{4,5}=6.2 \mathrm{~Hz}, \mathrm{H}-4\right), 4.76\left(\mathrm{ddd}, 1 \mathrm{H}, J_{2,3}=3.7\right.$ and $\left.J_{2,4}=0.6 \mathrm{~Hz}, \mathrm{H}-2\right), 4.53(\mathrm{~m}, 1 \mathrm{H}$, $J_{2^{\prime} \mathrm{ax}, 3^{\prime}}=11.0, J_{2^{\prime} \mathrm{eq}, 3^{\prime}}=5.5, J_{3^{\prime}, 4^{\prime}}=3.0$ and $\left.J_{3^{\prime}, \mathrm{NH}}=8.1 \mathrm{~Hz}, \mathrm{H}-3^{\prime}\right), 4.17\left(\mathrm{q}, 1 \mathrm{H}, J_{5^{\prime}, 6^{\prime}}=6.6 \mathrm{~Hz}, \mathrm{H}-5^{\prime}\right)$, $4.15\left(\mathrm{q}, 1 \mathrm{H}, J_{5,6}=6.7 \mathrm{~Hz}, \mathrm{H}-5\right), 4.13(\mathrm{~m}, 1 \mathrm{H}, \mathrm{H}-3), 2.18\left(\mathrm{~s}, 3 \mathrm{H}, \mathrm{CH}_{3} \mathrm{CO}\right), 2.06\left(\mathrm{~s}, 3 \mathrm{H}, \mathrm{CH}_{3} \mathrm{CO}\right), 1.90(\mathrm{~m}$, $2 \mathrm{H}, \mathrm{H}-2^{\prime} \mathrm{ax}$ and $\left.\mathrm{H}-2^{\prime} \mathrm{eq}\right), 1.34\left(\mathrm{~d}, 3 \mathrm{H}, \mathrm{CH}_{3}\right), 1.13\left(\mathrm{~d}, 3 \mathrm{H}, \mathrm{CH}_{3}{ }^{\prime}\right) ;{ }^{13} \mathrm{C} \mathrm{NMR}\left(\mathrm{CDCl}_{3}\right): \delta 170.8\left(\mathrm{CH}_{3} \mathrm{CO}\right)$, $169.7\left(\mathrm{CH}_{3} \mathrm{CO}\right), 144.7(\mathrm{C}-1), 99.8(\mathrm{C}-2), 96.6\left(\mathrm{C}^{-} \mathrm{I}^{\prime}\right), 72.9,72.2,70.9,70.3$ and $65.4\left(\mathrm{C}-3, \mathrm{C}-4, \mathrm{C}-5, \mathrm{C}-4^{\prime}\right.$

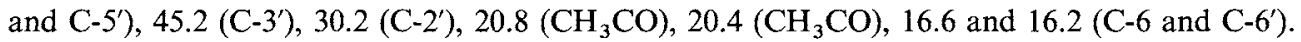

Anal Calcd for $\mathrm{C}_{18} \mathrm{H}_{24} \mathrm{~F}_{3} \mathrm{NO}_{8}$ : C 49.20, $\mathrm{H} 5.51, \mathrm{~N} 3.19$.

Found:

C $49.46, \mathrm{H} 5.54, \mathrm{~N} 3.13$.

Attempted Conversion of Disaccharide Glycal Derivatives 3 and 9 into Methyl 2-Deoxyglycosides by Direct Halogenation-glycosidation

4-O-(4-O-Acetyl-2,3,6-trideoxy-3-trifluoroacetamido- $\alpha$-L-lyxo-hexopyranosyl)-3- $O$-acetyl-1,5-anhydro-2,6-dideoxy-L-arabino-hex-1-enitol (3) $(0.162 \mathrm{~g}, 0.37 \mathrm{mmol})$ was dissolved in dry benzene $(10 \mathrm{ml})$ and the solution evaporated to half its volume. $\mathrm{HCl}$ gas was then bubbled through the magnetically stirred solution for 10 minutes at $5^{\circ} \mathrm{C}$. After 5 minutes at room temperature, the solvent was evaporated. The residue was redissolved in dry $\mathrm{CH}_{2} \mathrm{Cl}_{2}$, the solution evaporated, and the residue redissolved again.

At the same time, $\mathrm{HgO}(0.23 \mathrm{~g}, 1.07 \mathrm{mmol}), \mathrm{HgBr}_{2}(0.15 \mathrm{~g}, 0.41 \mathrm{mmol})$ and powdered $4 \AA$ molecular sieves $(0.36 \mathrm{~g})$ were mixed in dry $\mathrm{CH}_{2} \mathrm{Cl}_{2}(7 \mathrm{ml})$ and stirred magnetically for 1 hour at room temperature. To this mixture were succesively added dry $\mathrm{MeOH}(30 \mu 1,0.76 \mathrm{mmol})$ and then the previously prepared solution. The mixture was magnetically stirred at room temperature with TLC monitoring (2:1 hexane-EtOAc). After 2 hours the mixture was filtered through Celite and the filtrate was successively washed with $30 \%$ aqueous $\mathrm{KI}$, twice with water, and then dried $\left(\mathrm{Na}_{2} \mathrm{SO}_{4}\right)$ and evaporated. The resultant syrup ( $\sim 30 \mathrm{mg}$ ) showed by TLC, one major product. Purification by a small column chromatography $\left(5: 1\right.$ hexane-EtOAc) afforded pure $4(20 \mathrm{mg}, 0.07 \mathrm{mmol}, 18 \%)$; mp $167 \sim 169^{\circ} \mathrm{C}$, lit. $^{18)} 170^{\circ} \mathrm{C} ;[\alpha]_{\mathrm{D}}^{20}-20^{\circ}$ $\left(c 1.0, \mathrm{CHCl}_{3}\right)$, lit. ${ }^{18)}-22^{\circ}$.

Comparable results were observed when 4-O-acetyl-3-O-(4-O-acetyl-2,3,6-trideoxy-3-trifluoroacetamido- $\alpha$-L-lyxo-hexopyranosyl)-1,5-anhydro-2,6-dideoxy-L-arabino-hex-1-enitol (9) was used as the starting material.

Methyl 3-O-Acetyl-4-O-(4-O-acetyl-2,3,6-trideoxy-3-trifluoroacetamido- $\alpha$-L-lyxo-hexopyranosyl)-2,6dideoxy-2-iodo- $\alpha$-L-manno- (5) and $\beta$-L-gluco- (6) pyranosides

To a solution of glycal derivative $3(0.25 \mathrm{~g}, 0.57 \mathrm{mmol})$ and dry $\mathrm{MeOH}(0.1 \mathrm{ml}, 2.5 \mathrm{mmol})$ in dry $\mathrm{CH}_{3} \mathrm{CN}(3 \mathrm{ml})$, was added NIS $(0.200 \mathrm{~g}, 0.89 \mathrm{mmol})$ and the reaction was monitored by TLC $(2: 1$ hexane - EtOAc). After 2 hours the solution was diluted with $\mathrm{CH}_{2} \mathrm{Cl}_{2}$, washed with $10 \%$ aqueous $\mathrm{Na}_{2} \mathrm{~S}_{2} \mathrm{O}_{3}$, and finally with water, dried $\left(\mathrm{Na}_{2} \mathrm{SO}_{4}\right)$, and evaporated to afford a syrup that was purified by column chromatography $(5: 1$ hexane - EtOAc). Two pure fractions were isolated. The first one was an amorphous solid $(181 \mathrm{mg}, 53 \%)$ that characterized as the $\alpha$-L-manno isomer $5 ;[\alpha]_{\mathrm{D}}^{20}-76.3^{\circ}\left(c 1.0, \mathrm{CHCl}_{3}\right) ;{ }^{1} \mathrm{H} \mathrm{NMR}$ $\left(\mathrm{CDCl}_{3}\right): \delta 6.29\left(\mathrm{~d}, 1 \mathrm{H}, J_{\mathrm{NH}-3^{\prime}}=6.9 \mathrm{~Hz}, \mathrm{~N}-\mathrm{H}\right), 5.24\left(\mathrm{~d}, 1 \mathrm{H}, J_{1^{\prime}, 2^{\prime} \mathrm{ax}}=3.6 \mathrm{~Hz}, \mathrm{H}-1^{\prime}\right), 5.10\left(\mathrm{br} \mathrm{s}, 1 \mathrm{H}, \mathrm{H}^{\prime} 4^{\prime}\right), 4.96$ (br s, $1 \mathrm{H}, \mathrm{H}-1), 4.56\left(\mathrm{dd}, 1 \mathrm{H}, J_{1,2}=1.4\right.$ and $\left.J_{2,3}=4.2 \mathrm{~Hz}, \mathrm{H}-2\right), 4.49\left(\mathrm{dd}, 1 \mathrm{H}, J_{3,4}=8.5 \mathrm{~Hz}, \mathrm{H}-3\right), 4.48(\mathrm{~m}$, $\left.1 \mathrm{H}, \mathrm{H}-3^{\prime}\right), 4.16\left(\mathrm{q}, 1 \mathrm{H}, J_{5^{\prime}, 6^{\prime}}=6.5 \mathrm{~Hz}, \mathrm{H}-5^{\prime}\right), 3.86\left(\mathrm{~m}, 1 \mathrm{H}, J_{5,6}=6.2 \mathrm{~Hz}, \mathrm{H}-5\right), 3.75\left(\mathrm{t}, 1 \mathrm{H}, J_{4,5}=8.9 \mathrm{~Hz}\right.$, $\mathrm{H}-4), 3.39\left(\mathrm{~s}, 3 \mathrm{H}, \mathrm{OCH}_{3}\right), 2.17\left(\mathrm{~s}, 3 \mathrm{H}, \mathrm{CH}_{3} \mathrm{CO}\right), 2.10\left(\mathrm{~s}, 3 \mathrm{H}, \mathrm{CH}_{3} \mathrm{CO}\right), 1.93\left(\mathrm{td}, 1 \mathrm{H}, J_{2^{\prime} \mathrm{ax}, 2^{\prime} \mathrm{eq}}=12.5\right.$ and $\left.J_{2^{\prime} \text { ax. } 3^{\prime}}=12.5 \mathrm{~Hz}, \mathrm{H}-2^{\prime} \mathrm{ax}\right), 1.87\left(\mathrm{dd}, 1 \mathrm{H}, J_{2^{\prime} \mathrm{eq}, 3^{\prime}}=3.6 \mathrm{~Hz}, \mathrm{H}-2^{\prime} \mathrm{eq}\right), 1.35\left(\mathrm{~d}, 3 \mathrm{H}, \mathrm{CH}_{3}\right), 1.13\left(\mathrm{~d}, 3 \mathrm{H}, \mathrm{CH}_{3}{ }^{\prime}\right.$ ); ${ }^{13} \mathrm{C} \mathrm{NMR}\left(\mathrm{CDCl}_{3}\right): \delta 170.9\left(\mathrm{CH}_{3} \mathrm{CO}\right), 168.4\left(\mathrm{CH}_{3} \mathrm{CO}\right), 102.1(\mathrm{C}-1), 97.9\left(\mathrm{C}-1^{\prime}\right), 79.0(\mathrm{C}-4) .72 .4(\mathrm{C}-3)$, $\left.70.1\left(\mathrm{C}-4^{\prime}\right), 67.2(\mathrm{C}-5), 65.9\left({\mathrm{C}-5^{\prime}}^{\prime}\right), 55.1\left(\mathrm{OCH}_{3}\right), 45.1\left(\mathrm{C}^{\prime}\right)^{\prime}\right), 30.5(\mathrm{C}-2), 29.9\left(\mathrm{C}-2^{\prime}\right), 20.9\left(\mathrm{CH}_{3} \mathrm{CO}\right), 20.5$ $\left(\mathrm{CH}_{3} \mathrm{CO}\right), 18.1(\mathrm{C}-6), 16.7\left(\mathrm{C}-6^{\prime}\right)$

Anal Calcd for $\mathrm{C}_{19} \mathrm{H}_{27} \mathrm{~F}_{3} \mathrm{INO}_{9}$ : C 38.20, H 4.56, I 21.24, N 2.34. Found: 
The second fraction was isolated as a solid $(48 \mathrm{mg}, 14 \%)$ which crystallized from EtOH and was characterized as the $\beta$-L-gluco isomer 6; $\mathrm{mp} 200 \sim 202^{\circ} \mathrm{C} ;[\alpha]_{D}^{20}-89.6^{\circ}\left(c 1.0, \mathrm{CHCl}_{3}\right) ;{ }^{1} \mathrm{H} \mathrm{NMR}\left(\mathrm{CDCl}_{3}\right)$ : $\delta 6.19\left(\mathrm{~d}, 1 \mathrm{H}, J_{\mathrm{NH}-3^{\prime}}=6.2 \mathrm{~Hz}, \mathrm{~N}-\mathrm{H}\right), 5.28\left(\mathrm{dd}, 1 \mathrm{H}, J_{3,4}=9.0 \mathrm{~Hz}, \mathrm{H}-3\right), 5.12\left(\mathrm{br} \mathrm{s}, 1 \mathrm{H}, \mathrm{H}-4^{\prime}\right), 5.07(\mathrm{~d}, 1 \mathrm{H}$, $\left.J_{1^{\prime}, 2^{\prime} \mathrm{ax}}=3.8 \mathrm{~Hz}, \mathrm{H}-1^{\prime}\right), 4.47\left(\mathrm{~d}, 1 \mathrm{H}, J_{1,2}=8.9 \mathrm{~Hz}, \mathrm{H}-1\right), 4.43\left(\mathrm{dq}, 1 \mathrm{H}, J_{2^{\prime} \mathrm{ax} .3^{\prime}}=14.0 \mathrm{~Hz}, \mathrm{H}-3^{\prime}\right), 4.13(\mathrm{q}, 1 \mathrm{H}$, $\left.J_{5^{\prime}, 6^{\prime}}=6.5 \mathrm{~Hz}, \mathrm{H}-5^{\prime}\right), 3.80\left(\mathrm{dd}, 1 \mathrm{H}, J_{2,3}=11.2 \mathrm{~Hz}, \mathrm{H}-2\right), 3.55\left(\mathrm{~s}, 3 \mathrm{H}, \mathrm{OCH}_{3}\right), 3.53\left(\mathrm{~m}, 1 \mathrm{H}, J_{5,6}=6.1 \mathrm{~Hz}\right.$, $\mathrm{H}-5), 3.44\left(\mathrm{t}, 1 \mathrm{H}, J_{4,5}=9.0 \mathrm{~Hz}, \mathrm{H}-4\right), 2.16\left(\mathrm{~s}, 3 \mathrm{H}, \mathrm{CH}_{3} \mathrm{CO}\right), 2.13\left(\mathrm{~s}, 3 \mathrm{H}, \mathrm{CH}_{3} \mathrm{CO}\right), 1.89(\mathrm{td}, 1 \mathrm{H}$, $\left.J_{2^{\prime} \mathbf{a x}, 2^{\prime} \text { eq }}=12.7 \mathrm{~Hz}, \mathrm{H}-2^{\prime} \mathrm{ax}\right), 1.78\left(\mathrm{dd}, 1 \mathrm{H}, J_{2^{\prime} \mathrm{eq}, 3^{\prime}}=5.0 \mathrm{~Hz}, \mathrm{H}-2^{\prime} \mathrm{eq}\right), 1.36\left(\mathrm{~d}, 3 \mathrm{H}, \mathrm{CH}_{3}\right), 1.10\left(\mathrm{~d}, 3 \mathrm{H}, \mathrm{CH}_{3}{ }^{\prime}\right)$; ${ }^{13} \mathrm{C}$ NMR $\left(\mathrm{CDCl}_{3}\right): \delta 170.7\left(\mathrm{CH}_{3} \mathrm{CO}\right), 169.4\left(\mathrm{CH}_{3} \mathrm{CO}\right), 103.6(\mathrm{C}-1), 97.6\left(\mathrm{C}-1^{\prime}\right), 80.8,77.9,70.9,69.7$ and $66.0\left(\mathrm{C}-3, \mathrm{C}-4, \mathrm{C}-5, \mathrm{C}-4^{\prime}\right.$ and $\left.\mathrm{C}-5^{\prime}\right), 57.5\left(\mathrm{OCH}_{3}\right), 44.9\left(\mathrm{C}-3^{\prime}\right), 29.8$ and $29.7\left(\mathrm{C}-2\right.$ and $\left.\mathrm{C}-2^{\prime}\right), 21.2$ $\left(\mathrm{CH}_{3} \mathrm{CO}\right), 20.5\left(\mathrm{CH}_{3} \mathrm{CO}\right), 18.0$ and $16.7\left(\mathrm{C}-6\right.$ and $\left.\mathrm{C}-6{ }^{\prime}\right)$.

Anal Calcd for $\mathrm{C}_{19} \mathrm{H}_{27} \mathrm{~F}_{3} \mathrm{INO}_{9}$ : C 38.20, H 4.56, I 21.24, N 2.34 . Found:

C $38.29, \mathrm{H} 4.57$, I $21.30, \mathrm{~N} 2.27$.

Methyl 4-O-Acetyl-3-O-(4-O-acetyl-2,3,6-trideoxy-3-trifluoroacetamido- $\alpha$-L-lyxo-hexopyranosyl)-2,6dideoxy-2-iodo- $\alpha$-L-manno- (10) and $\beta$-L-gluco- (11) pyranosides

The procedure just described was applied with the glycal derivative 9. Column chromatographic purification ( $5: 1$ hexane - EtOAc) afforded two fractions. The first fraction was obtained as a solid (176 mg, $52 \%$ ) that crystallized from EtOH and was characterized as the $\alpha$-L-manno isomer 10; $\mathrm{mp} 218 \sim 220^{\circ} \mathrm{C}$; $[\alpha]_{\mathrm{D}}^{20}-73.1^{\circ}\left(c 1.0, \mathrm{CHCl}_{3}\right) ;{ }^{1} \mathrm{H} \mathrm{NMR}\left(\mathrm{CDCl}_{3}\right): \delta 6.32\left(\mathrm{~d}, 1 \mathrm{H}, J_{\mathrm{NH}-3^{\prime}}=6.4 \mathrm{~Hz}, \mathrm{~N}-\mathrm{H}\right), 5.13\left(\mathrm{br} \mathrm{s}, 1 \mathrm{H}, \mathrm{H}-4^{\prime}\right)$, $5.09\left(\mathrm{~d}, 1 \mathrm{H}, J_{1^{\prime}, 2^{\prime} \mathbf{a x}}=3.6 \mathrm{~Hz}, \mathrm{H}-1^{\prime}\right), 5.06\left(\mathrm{t}, 1 \mathrm{H}, J_{4,5}=9.5 \mathrm{~Hz}, \mathrm{H}-4\right), 5.00(\mathrm{~s}, 1 \mathrm{H}, \mathrm{H}-1), 4.49\left(\mathrm{~m}, 1 \mathrm{H}, \mathrm{H}-3^{\prime}\right)$, $4.35\left(\mathrm{~m}, 2 \mathrm{H}, \mathrm{H}-2\right.$ and $\left.\mathrm{H}-5^{\prime}\right), 3.84\left(\mathrm{~m}, 1 \mathrm{H}, J_{5,6}=6.3 \mathrm{~Hz}, \mathrm{H}-5\right), 3.42\left(\mathrm{dd}, 1 \mathrm{H}, J_{2,3}=4.2\right.$ and $J_{3,4}=8.5 \mathrm{~Hz}$, $\mathrm{H}-3), 3.37$ (s, $\left.3 \mathrm{H}, \mathrm{OCH}_{3}\right), 2.17\left(\mathrm{~s}, 3 \mathrm{H}, \mathrm{CH}_{3} \mathrm{CO}\right), 2.08\left(\mathrm{~s}, 3 \mathrm{H}, \mathrm{CH}_{3} \mathrm{CO}\right), 1.93\left(\mathrm{dd}, 1 \mathrm{H}, J_{2^{\prime} \text { eq, } 3^{\prime}}=5.1\right.$ and $\left.J_{2^{\prime} \text { eq }, 2^{\prime}{ }^{\prime} \mathrm{xx}}=12.8 \mathrm{~Hz}, \mathrm{H}-2^{\prime} \mathrm{eq}\right), 1.86\left(\mathrm{td}, 1 \mathrm{H}, J_{2^{\prime} \mathrm{ax}, 3^{\prime}}=12.5 \mathrm{~Hz}, \mathrm{H}-2^{\prime} \mathrm{ax}\right), 1.22\left(\mathrm{~d}, 3 \mathrm{H}, \mathrm{CH}_{3}\right), 1.12\left(\mathrm{~d}, 3 \mathrm{H}, \mathrm{CH}_{3}{ }^{\prime}\right)$; ${ }^{13} \mathrm{C} \mathrm{NMR}\left(\mathrm{CDCl}_{3}\right): \delta 171.1\left(\mathrm{CH}_{3} \mathrm{CO}\right), 169.7\left(\mathrm{CH}_{3} \mathrm{CO}\right), 102.5(\mathrm{C}-1), 98.8\left(\mathrm{C}-1^{\prime}\right), 75.3(\mathrm{C}-3), 74.4(\mathrm{C}-4)$, $70.2\left(\mathrm{C}-4^{\prime}\right), 67.2(\mathrm{C}-5), 66.9\left({\mathrm{C}-5^{\prime}}^{\prime}\right), 55.2\left(\mathrm{OCH}_{3}\right), 45.7\left(\mathrm{C}-3^{\prime}\right), 33.4(\mathrm{C}-2), 30.2\left(\mathrm{C}-2^{\prime}\right), 20.8\left(\mathrm{CH}_{3} \mathrm{CO}\right), 20.6$ $\left(\mathrm{CH}_{3} \mathrm{CO}\right), 17.6(\mathrm{C}-6), 16.7\left(\mathrm{C}-6^{\prime}\right)$.

Anal Calcd for $\mathrm{C}_{19} \mathrm{H}_{27} \mathrm{~F}_{3} \mathrm{INO}_{9}$ : C $38.20, \mathrm{H} 4.56, \mathrm{I} 21.24, \mathrm{~N} 2.34$.

Found:

C $38.28, \mathrm{H} 4.58$, I $21.18, \mathrm{~N} 2.30$.

The second fraction was isolated as a solid $(54 \mathrm{mg}, 16 \%)$ which crystallized from EtOH and was characterized as the $\beta$-L-gluco isomer 11; $\mathrm{mp} 215 \sim 217^{\circ} \mathrm{C} ;[\alpha]_{\mathrm{D}}^{20}-148^{\circ}\left(c 1.0, \mathrm{CHCl}_{3}\right) ;{ }^{1} \mathrm{H} \mathrm{NMR}\left(\mathrm{CDCl}_{3}\right)$ : $\delta 6.20\left(\mathrm{~d}, 1 \mathrm{H}, J_{\mathrm{NH}-3^{\prime}}=7.2 \mathrm{~Hz}, \mathrm{~N}-\mathrm{H}\right), 5.17\left(\mathrm{~m}, 2 \mathrm{H}, \mathrm{H}-\mathrm{1}^{\prime}\right.$ and $\left.\mathrm{H}-4^{\prime}\right), 4.78\left(\mathrm{t}, 1 \mathrm{H}, J_{4,5}=9.0 \mathrm{~Hz}, \mathrm{H}-4\right), 4.74(\mathrm{q}$, $\left.1 \mathrm{H}, J_{5^{\prime}, 6^{\prime}}=6.5 \mathrm{~Hz}, \mathrm{H}-5^{\prime}\right), 4.55\left(\mathrm{~m}, 1 \mathrm{H}, \mathrm{H}-3^{\prime}\right), 4.48\left(\mathrm{~d}, 1 \mathrm{H}, J_{1,2}=9.0 \mathrm{~Hz}, \mathrm{H}-1\right), 3.95\left(\mathrm{dd}, 1 \mathrm{H}, J_{3,4}=8.7 \mathrm{~Hz}\right.$, $\mathrm{H}-3), 3.81\left(\mathrm{dd}, 1 \mathrm{H}, J_{2,3}=10.5 \mathrm{~Hz}, \mathrm{H}-2\right), 3.54\left(\mathrm{~s}, 3 \mathrm{H}, \mathrm{OCH}_{3}\right), 3.48\left(\mathrm{~m}, 1 \mathrm{H}, J_{5,6}=6.2 \mathrm{~Hz}, \mathrm{H}-5\right), 2.16(\mathrm{~s}, 3 \mathrm{H}$, $\left.\mathrm{CH}_{3} \mathrm{CO}\right), 2.08\left(\mathrm{~s}, 3 \mathrm{H}, \mathrm{CH}_{3} \mathrm{CO}\right), 1.89\left(\mathrm{td}, 1 \mathrm{H}, J_{2^{\prime} \mathrm{ax}, 2^{\prime} \mathrm{eq}}=12.5\right.$ and $\left.J_{2^{\prime} \mathrm{ax}, 3^{\prime}}=13.5 \mathrm{~Hz}, \mathrm{H}-2^{\prime} \mathrm{ax}\right), 1.81(\mathrm{dd}, 1 \mathrm{H}$, $\left.J_{2^{\prime} \mathrm{eq}, 3^{\prime}}=4.8 \mathrm{~Hz}, \mathrm{H}-2^{\prime} \mathrm{eq}\right), 1.20\left(\mathrm{~d}, 3 \mathrm{H}, \mathrm{CH}_{3}\right), 1.12\left(\mathrm{~d}, 3 \mathrm{H}, \mathrm{CH}_{3}{ }^{\prime}\right) ;{ }^{13} \mathrm{C} \mathrm{NMR}\left(\mathrm{CDCl}_{3}\right): \delta 170.9\left(\mathrm{CH}_{3} \mathrm{CO}\right)$, $169.4\left(\mathrm{CH}_{3} \mathrm{CO}\right), 104.1(\mathrm{C}-1), 97.2\left(\mathrm{C}-1^{\prime}\right), 81.2(\mathrm{C}-3), 77.2(\mathrm{C}-4), 70.4\left(\mathrm{C}-4^{\prime}\right), 70.0(\mathrm{C}-5), 66.6\left(\mathrm{C}-5^{\prime}\right), 57.3$ $\left(\mathrm{OCH}_{3}\right), 45.1\left(\mathrm{C}-3^{\prime}\right), 31.5(\mathrm{C}-2), 30.3\left(\mathrm{C}-2^{\prime}\right), 20.9\left(\mathrm{CH}_{3} \mathrm{CO}\right), 20.6\left(\mathrm{CH}_{3} \mathrm{CO}\right), 17.2(\mathrm{C}-6), 16.6\left(\mathrm{C}-6^{\prime}\right)$.

Anal Calcd for $\mathrm{C}_{19} \mathrm{H}_{27} \mathrm{~F}_{3} \mathrm{INO}_{9}$ : C $38.20, \mathrm{H} 4.56, \mathrm{I} 21.24, \mathrm{~N} 2.34$.

Found:

C $38.25, \mathrm{H} 4.61$, I $21.31, \mathrm{~N} 2.33$.

Methyl 3-O-Acetyl-4-O-(4-O-acetyl-2,3,6-trideoxy-3-trifluoroacetamido- $\alpha$-L-lyxo-hexopyranosyl)-2,6dideoxy- $\alpha$-L-arabino-hexopyranoside (7)

To a solution of the $\alpha$-L-manno derivative $5(100 \mathrm{mg}, 0.17 \mathrm{mmol})$ in dry benzene ( $1 \mathrm{ml})$ was added $\mathrm{Bu}_{3} \mathrm{SnH}(50 \mu \mathrm{l}, 0.2 \mathrm{mmol})$ and a few $\mathrm{mg}$ of azobis (isobutyronitrile) (AIBN) in an inert atmosphere and the mixture was heated at $60^{\circ} \mathrm{C}$. The reaction was complete after 2 hours (TLC). The mixture was diluted with $\mathrm{CH}_{3} \mathrm{CN}$ and washed three times with hexane ${ }^{19}$. The solution was evaporated to a syrup that crystallized from EtOH - hexane to afford pure $7(58 \mathrm{mg}, 73 \%)$; mp $115 \sim 117^{\circ} \mathrm{C} ;[\alpha]_{\mathrm{D}}^{20}-172^{\circ}\left(c 1.0, \mathrm{CHCl}_{3}\right)$; ${ }^{1} \mathrm{H} \mathrm{NMR}\left(\mathrm{CDCl}_{3}\right): \delta 6.23\left(\mathrm{~d}, 1 \mathrm{H}, J_{\mathrm{NH}-3^{\prime}}=7.1 \mathrm{~Hz}, \mathrm{~N}-\mathrm{H}\right), 5.25\left(\mathrm{~d}, 1 \mathrm{H}, J_{1^{\prime}, 2^{\prime} \mathrm{ax}}=3.7 \mathrm{~Hz}, \mathrm{H}-1^{\prime}\right), 5.18(\mathrm{dq}, 1 \mathrm{H}$, $\left.J_{3,4}=9.1 \mathrm{~Hz}, \mathrm{H}-3\right), 5.12\left(\mathrm{brs}, 1 \mathrm{H}, \mathrm{H}-4^{\prime}\right), 4.70\left(\mathrm{~d}, 1 \mathrm{H}, J_{1,2 \mathrm{ax}}=3.7 \mathrm{~Hz}, \mathrm{H}-1\right), 4.48\left(\mathrm{~m}, 1 \mathrm{H}, \mathrm{H}-3^{\prime}\right), 4.17(\mathrm{q}, 1 \mathrm{H}$, $\left.J_{5^{\prime}, 6^{\prime}}=6.6 \mathrm{~Hz}, \mathrm{H}-5^{\prime}\right), 3.77\left(\mathrm{~m}, 1 \mathrm{H}, J_{5,6}=6.2 \mathrm{~Hz}, \mathrm{H}-5\right), 3.41\left(\mathrm{t}, 1 \mathrm{H}, J_{4,5}=9.1 \mathrm{~Hz}, \mathrm{H}-4\right), 3.33\left(\mathrm{~s}, 3 \mathrm{H}, \mathrm{OCH}_{3}\right)$, $2.24\left(\mathrm{dd}, 1 \mathrm{H}, J_{2 \mathrm{eq}, 3}=5.0\right.$ and $\left.J_{2 \mathrm{eq}, 2 \mathrm{ax}}=12.8 \mathrm{~Hz}, \mathrm{H}-2 \mathrm{eq}\right), 2.17\left(\mathrm{~s}, 3 \mathrm{H}, \mathrm{CH}_{3} \mathrm{CO}\right), 2.03\left(\mathrm{~s}, 3 \mathrm{H}, \mathrm{CH}_{3} \mathrm{CO}\right), 1.91$ $\left(\mathrm{td}, 1 \mathrm{H}, J_{2^{\prime} \mathrm{ax}, 2^{\prime} \mathrm{eq}}=12.4\right.$ and $\left.J_{2^{\prime} \mathrm{ax}, 3^{\prime}}=12.5 \mathrm{~Hz}, \mathrm{H}-2^{\prime} \mathrm{ax}\right), 1.86\left(\mathrm{dd}, 1 \mathrm{H}, J_{2^{\prime} \mathrm{eq}, 3^{\prime}}=5.1 \mathrm{~Hz}, \mathrm{H}-2^{\prime} \mathrm{eq}\right), 1.66(\mathrm{td}, 1 \mathrm{H}$, 
$\left.J_{2 \mathrm{ax} .3}=11.3 \mathrm{~Hz}, \mathrm{H}-2 \mathrm{ax}\right), 1.30\left(\mathrm{~d}, 3 \mathrm{H}, \mathrm{CH}_{3}\right), 1.12\left(\mathrm{~d}, 3 \mathrm{H}, \mathrm{CH}_{3}{ }^{\prime}\right) ;{ }^{13} \mathrm{C} \mathrm{NMR}\left(\mathrm{CDCl}_{3}\right): \delta 170.9\left(\mathrm{CH}_{3} \mathrm{CO}\right)$, $169.8\left(\mathrm{CH}_{3} \mathrm{CO}\right), 97.7(\mathrm{C}-1), 97.5\left(\mathrm{C}-1^{\prime}\right), 80.5(\mathrm{C}-4), 72.6(\mathrm{C}-3), 70.2\left(\mathrm{C}-4^{\prime}\right), 66.1(\mathrm{C}-5), 65.8\left(\mathrm{C}-5^{\prime}\right), 54.7$ $\left(\mathrm{OCH}_{3}\right), 45.2\left(\mathrm{C}-3^{\prime}\right), 32.6(\mathrm{C}-2), 30.2\left(\mathrm{C}^{\prime}\right), 21.2\left(\mathrm{CH}_{3} \mathrm{CO}\right), 20.6\left(\mathrm{CH}_{3} \mathrm{CO}\right), 18.4(\mathrm{C}-6), 16.8\left(\mathrm{C}-6^{\prime}\right)$.

Anal Calcd for $\mathrm{C}_{19} \mathrm{H}_{28} \mathrm{~F}_{3} \mathrm{NO}_{9}$ : C $48.41, \mathrm{H} 5.99, \mathrm{~N} 2.97$.

Found:

C 48.48, H 5.99 , N 2.92 .

Methyl 4-O-Acetyl-3-O-(4-O-acetyl-2,3,6-trideoxy-3-trifluoroacetamido- $\alpha$-L-lyxo-hexopyranosyl)-2,6dideoxy- $\alpha$-L-arabino-hexopyranoside (12)

The conditions just described were applied to with the $\alpha$-L-manno derivative 10. Similar processing gave a solid that crystallized from EtOH to afford pure $12(60 \mathrm{mg}, 76 \%) ; \mathrm{mp} 248 \sim 250^{\circ} \mathrm{C}$; $[\alpha]_{\mathrm{D}}^{20}-175.3^{\circ}(c 1.0$, $\left.\mathrm{CHCl}_{3}\right) ;{ }^{1} \mathrm{H} \mathrm{NMR}\left(\mathrm{CDCl}_{3}\right): \delta 6.18\left(\mathrm{~d}, 1 \mathrm{H}, J_{\mathrm{NH}-3^{\prime}}=7.0 \mathrm{~Hz}, \mathrm{~N}-\mathrm{H}\right), 5.04$ (brs, $\left.1 \mathrm{H}, \mathrm{H}-4^{\prime}\right), 5.09$ (brs, $1 \mathrm{H}$, $\left.\mathrm{H}^{-1} \mathrm{1}^{\prime}\right), 4.74\left(\mathrm{~d}, 1 \mathrm{H}, J_{1,2 \mathrm{ax}}=3.1 \mathrm{~Hz}, \mathrm{H}-1\right), 4.70\left(\mathrm{t}, 1 \mathrm{H}, J_{3,4}=9.3 \mathrm{~Hz}, \mathrm{H}-4\right), 4.48\left(\mathrm{~m}, 1 \mathrm{H}, \mathrm{H}-3^{\prime}\right), 4.10(\mathrm{q}, 1 \mathrm{H}$, $\left.J_{5^{\prime}, 6^{\prime}}=6.5 \mathrm{~Hz}, \mathrm{H}-5^{\prime}\right), 4.02\left(\mathrm{dq}, 1 \mathrm{H}, J_{2 \mathrm{ax}, 3}=11.5\right.$ and $\left.J_{2 \mathrm{eq}, 3}=5.3 \mathrm{~Hz}, \mathrm{H}-3\right), 3.74\left(\mathrm{~m}, 1 \mathrm{H}, J_{4,5}=9.5 \mathrm{~Hz}, \mathrm{H}-5\right)$, $3.32\left(\mathrm{~s}, 3 \mathrm{H}, \mathrm{OCH}_{3}\right), 2.16$ (s and $\mathrm{m}, 4 \mathrm{H}, \mathrm{CH}_{3} \mathrm{CO}$ and $\left.\mathrm{H}-2 \mathrm{eq}\right), 2.07\left(\mathrm{~s}, 3 \mathrm{H}, \mathrm{CH}_{3} \mathrm{CO}\right), 1.90 \sim 1.77(\mathrm{~m}, 3 \mathrm{H}$, $\mathrm{H}-2 \mathrm{ax}, \mathrm{H}-2^{\prime} \mathrm{eq}$ and $\left.\mathrm{H}-2^{\prime} \mathrm{ax}\right), 1.16\left(\mathrm{~d}, 3 \mathrm{H}, \mathrm{CH}_{3}\right), 1.10\left(\mathrm{~d}, 3 \mathrm{H}, \mathrm{CH}_{3}{ }^{\prime}\right) ;{ }^{13} \mathrm{C} \mathrm{NMR}\left(\mathrm{CDCl}_{3}\right): \delta 170.9\left(\mathrm{CH}_{3} \mathrm{CO}\right)$, $169.7\left(\mathrm{CH}_{3} \mathrm{CO}\right), 98.1(\mathrm{C}-1), 97.8\left(\mathrm{C}-1^{\prime}\right), 76.8(\mathrm{C}-4), 73.9(\mathrm{C}-3), 70.2\left(\mathrm{C}-4^{\prime}\right), 65.7(\mathrm{C}-5), 65.3\left(\mathrm{C}-5^{\prime}\right), 54.7$ $\left(\mathrm{OCH}_{3}\right), 45.2\left(\mathrm{C}-3^{\prime}\right), 36.9(\mathrm{C}-2), 30.2\left(\mathrm{C}^{\prime} 2^{\prime}\right), 20.8\left(\mathrm{CH}_{3} \mathrm{CO}\right), 20.6\left(\mathrm{CH}_{3} \mathrm{CO}\right), 17.6(\mathrm{C}-6), 16.7\left(\mathrm{C}-6^{\prime}\right)$.

Anal Calcd for $\mathrm{C}_{19} \mathrm{H}_{28} \mathrm{~F}_{3} \mathrm{NO}_{9}$ : Found:
C $48.41, \mathrm{H} 5.99, \mathrm{~N} 2.97$.

C 48.49, H 5.99, N 2.95 .

7-O-[4-O-Acetyl-3-O-(4-O-acetyl-2,3,6-trideoxy-3-trifluoroacetamido- $\alpha$-L-lyxo-hexopyranosyl)-2,6dideoxy-2-iodo- $\alpha$-L-mannopyranosyl]-daunomycinone (13)

To a solution of glycal derivative $9(0.50 \mathrm{~g}, 1.14 \mathrm{mmol})$ and daunomycinone $(0.44 \mathrm{~g}, 1.10 \mathrm{mmol})$ in dry $\mathrm{CH}_{3} \mathrm{CN}(8.7 \mathrm{ml})$ and dry THF $(3.6 \mathrm{ml})$, was added NIS $(0.37 \mathrm{~g}, 1.64 \mathrm{mmol})$ and the mixture was stirred magnetically at room temperature with TLC monitoring ( $3: 1$ toluene-acetone). Some daunomycinone still remained unreacted after 12 hours, and more $9(0.25 \mathrm{~g}, 0.57 \mathrm{mmol})$ and NIS $(0.19 \mathrm{~g}, 0.82 \mathrm{mmol})$ were added. After a further 2 hours the reaction was complete and the solution was diluted with $\mathrm{CH}_{2} \mathrm{Cl}_{2}(50 \mathrm{ml})$. The resulting solution was washed with a $10 \%$ aqueous $\mathrm{Na}_{2} \mathrm{~S}_{2} \mathrm{O}_{3}(2 \times 30 \mathrm{ml})$ and water $(2 \times 30 \mathrm{ml})$, dried $\left(\mathrm{Na}_{2} \mathrm{SO}_{4}\right)$, and evaporated to afford a red solid which was purified by column chromatography $(50 \mathrm{~g}$ of silica gel, $8: 1$ toluene-acetone). The chromatographically major pure fraction was isolated as a red solid which crystallized from acetone - hexane to yield pure $13(53 \mathrm{mg}, 50 \%) ; \mathrm{mp} 170 \sim 172^{\circ} \mathrm{C} ;[\alpha]_{\mathrm{D}}^{20}+24.0^{\circ}$ (c $\left.1.0, \mathrm{CHCl}_{3}\right) ;{ }^{1} \mathrm{H}$ NMR $\left(\mathrm{CDCl}_{3}\right): \delta 14.01$ and $13.27(\mathrm{~s}, 2 \mathrm{H}, \mathrm{OH}-6$ and $\mathrm{OH}-11), 8.03$ (dd, $1 \mathrm{H}, J_{1,2}=7.8$ and $\left.J_{1,3}=0.8 \mathrm{~Hz}, \mathrm{H}-1\right), 7.80\left(\mathrm{t}, 1 \mathrm{H}, J_{2,3}=8.4 \mathrm{~Hz}, \mathrm{H}-2\right), 7.40(\mathrm{dd}, 1 \mathrm{H}, \mathrm{H}-3), 6.37\left(\mathrm{~d}, 1 \mathrm{H}, J_{\mathrm{NH}-3^{\prime \prime}}=6.8 \mathrm{~Hz}\right.$, $\mathrm{N}-\mathrm{H}), 5.75\left(\mathrm{~d}, 1 \mathrm{H}, \mathrm{H}-1^{\prime}\right), 5.24(\mathrm{~m}, 1 \mathrm{H}, \mathrm{H}-7), 5.10\left(\mathrm{t}, 1 \mathrm{H}, J_{3^{\prime}, 4^{\prime}}=9.3\right.$ and $\left.J_{4^{\prime}, 5^{\prime}}=9.3 \mathrm{~Hz}, \mathrm{H}-4^{\prime}\right), 5.07(\mathrm{brs}$, $\left.1 \mathrm{H}, \mathrm{H}-4^{\prime \prime}\right), 5.00\left(\mathrm{~d}, 1 \mathrm{H}, \mathrm{H}-1^{\prime \prime}\right), 4.46\left(\mathrm{~m}, 1 \mathrm{H}, \mathrm{H}-3^{\prime \prime}\right), 4.43\left(\mathrm{dd}, 1 \mathrm{H}, J_{1^{\prime}, 2^{\prime}}=1.4\right.$ and $\left.J_{2^{\prime}, 3^{\prime}}=4.0 \mathrm{~Hz}, \mathrm{H}-2^{\prime}\right), 4.36$ $\left(\mathrm{q}, 1 \mathrm{H}, J_{5^{\prime \prime}, 6^{\prime \prime}}=6.6 \mathrm{~Hz}, \mathrm{H}-5^{\prime \prime}\right), 4.15(\mathrm{~s}, 1 \mathrm{H}, \mathrm{OH}-9), 4.09\left(\mathrm{~s}, 3 \mathrm{H}, \mathrm{OCH}_{3}\right), 4.06\left(\mathrm{~m}, 1 \mathrm{H}, J_{4^{\prime}, 5^{\prime}}=9.6\right.$ and $\left.J_{5^{\prime}, 6^{\prime}}=6.3 \mathrm{~Hz}, \mathrm{H}-5^{\prime}\right), 3.23\left(\mathrm{dd}, 1 \mathrm{H}, J_{8 \mathrm{eq}, 10 \mathrm{eq}}=1.8 \mathrm{~Hz}, \mathrm{H}-10 \mathrm{eq}\right), 3.22\left(\mathrm{dd}, 1 \mathrm{H}, J_{3^{\prime}, 4^{\prime}}=8.8 \mathrm{~Hz}, \mathrm{H}-3^{\prime}\right), 2.94(\mathrm{~d}$, $\left.1 \mathrm{H}, J_{10 \mathrm{ax}, 10_{\mathrm{eq}}}=18.7 \mathrm{~Hz}, \mathrm{H}-10 \mathrm{ax}\right), 2.42\left(\mathrm{~s}, 3 \mathrm{H}, \mathrm{H}_{3}-14\right), 2.34$ (brd, $\left.1 \mathrm{H}, J_{8 \mathrm{ax}, 8 \mathrm{eq}}=15.0 \mathrm{~Hz}, \mathrm{H}-8 \mathrm{eq}\right), 2.16(\mathrm{dd}$, $\left.1 \mathrm{H}, J_{7,8 \mathrm{ax}}=4.4 \mathrm{~Hz}, \mathrm{H}-8 \mathrm{ax}\right), 2.12\left(\mathrm{~s}, 3 \mathrm{H}, \mathrm{CH}_{3} \mathrm{CO}\right), 2.09\left(\mathrm{~s}, 3 \mathrm{H}, \mathrm{CH}_{3} \mathrm{CO}\right), 1.90\left(\mathrm{dd}, 1 \mathrm{H}, J_{2}{ }^{\prime \prime}\right.$ eq, 3" $=4.6$ and $\left.J_{2^{\prime \prime} \text { eq, } 2^{\prime \prime a x}}=12.5 \mathrm{~Hz}, \mathrm{H}-2^{\prime \prime} \mathrm{eq}\right), 1.81\left(\mathrm{~m}, 1 \mathrm{H}, J_{1 ", 22^{\prime \prime a x}}=3.7\right.$ and $\left.J_{2 " \text { ax. } 3^{\prime \prime}}=12.8 \mathrm{~Hz}, \mathrm{H}-2^{\prime \prime} \mathrm{ax}\right), 1.27\left(\mathrm{~d}, 3 \mathrm{H}, \mathrm{CH}_{3}{ }^{\prime}\right)$, $1.04\left(\mathrm{~d}, 3 \mathrm{H}, \mathrm{CH}_{3}{ }^{\prime}\right) ;{ }^{13} \mathrm{C}$ NMR (acetone- $\left.d_{6}\right): \delta 211.67(\mathrm{C}-13), 187.27$ and $186.93(\mathrm{C}-5$ and $\mathrm{C}-12), 170.83$ $\left(\mathrm{CH}_{3} \mathrm{CO}\right), 170.13\left(\mathrm{CH}_{3} \mathrm{CO}\right), 161.87(\mathrm{C}-4), 156.97$ and $156.47(\mathrm{C}-6$ and $\mathrm{C}-11), 136.56,135.53$ and 134.85 (C-2, C-6a, C-10a and C-12a), 120.92, 119.95 and 119.71 (C-1, C-4a and C-3), 111.72 and 111.68 (C-5a and $\mathrm{C}-11 \mathrm{a}), 105.57$ and $99.48\left(\mathrm{C}-1^{\prime}\right.$ and $\left.\mathrm{C}-1^{\prime \prime}\right), 76.49,75.13,74.07,72.03,69.84,68.66$ and 67.07 (C-3', $\mathrm{C}-4^{\prime}, \mathrm{C}-4^{\prime \prime}, \mathrm{C}-5^{\prime \prime}, \mathrm{C}-5^{\prime}, \mathrm{C}-7$ and $\left.\mathrm{C}-9\right), 56.79\left(\mathrm{OCH}_{3}\right), 45.92\left(\mathrm{C}-3^{\prime \prime}\right), 36.33$ and $35.73(\mathrm{C}-8$ and $\mathrm{C}-10), 32.86$ $\left(\mathrm{C}-2^{\prime \prime}\right), 24.27(\mathrm{C}-14), 20.58\left(\mathrm{CH}_{3} \mathrm{CO}\right), 20.33\left(\mathrm{CH}_{3} \mathrm{CO}\right), 17.62$ and $16.76\left(\mathrm{C}-6^{\prime}\right.$ and $\left.\mathrm{C}-6^{\prime \prime}\right)$.

Anal Calcd for $\mathrm{C}_{39} \mathrm{H}_{41} \mathrm{~F}_{3} \mathrm{INO}_{16} \cdot \mathrm{H}_{2} \mathrm{O}:$ C $47.72, \mathrm{H} 4.41$, I 12.93, N 1.43 . Found:

C 47.76, H 4.49, I 12.90, N 1.37 .

7-O-[4-O-Acetyl-3-O-(4-O-acetyl-2,3,6-trideoxy-3-trifluoroacetamido- $\alpha$-L-lyxo-hexopyranosyl)-2,6dideoxy- $\alpha$-L-arabino-hexopyranosyl]-daunomycinone (14)

To a solution of $13(0.200 \mathrm{~g}, 0.21 \mathrm{mmol})$ in dry benzene $(6 \mathrm{ml})$, were added $\mathrm{Bu}_{3} \mathrm{SnH}(0.2 \mathrm{ml}, 0.8 \mathrm{mmol})$ and a few $\mathrm{mg}$ of AIBN in an inert atmosphere and the mixture was stirred magnetically at $45^{\circ} \mathrm{C}$ with TLC 
monitoring (6:1 $\mathrm{CHCl}_{3}$-acetone). After 48 hours the reaction was terminated by evaporation, and the remaining syrup was redissolved in $\mathrm{CH}_{3} \mathrm{CN}$ and washed with hexane ${ }^{19)}$. The acetonitrile solution was evaporated to afford a red solid which was purified by column chromatography (20 $\mathrm{g}$ silica gel, 15:1 $\mathrm{CHCl}_{3}$ - acetone). One pure fraction was isolated as a red solid, which crystallized from acetone - hexane to yield pure $14(90 \mathrm{mg}, 55 \%) ; \mathrm{mp} 264 \sim 266^{\circ} \mathrm{C} ;[\alpha]_{\mathrm{D}}^{20}+55.3^{\circ}\left(c 0.2, \mathrm{CHCl}_{3}\right) ;{ }^{1} \mathrm{H}$ NMR $\left(\mathrm{CDCl}_{3}\right): \delta 14.01$ and $13.29(\mathrm{~s}, 2 \mathrm{H}, \mathrm{OH}-6$ and $\mathrm{OH}-11), 8.04\left(\mathrm{~d}, 1 \mathrm{H}, J_{1.2}=7.8 \mathrm{~Hz}, \mathrm{H}-1\right), 7.78(\mathrm{t}, 1 \mathrm{H}, \mathrm{H}-2), 7.39(\mathrm{~d}, 1 \mathrm{H}$, $\left.J_{2,3}=8.2 \mathrm{~Hz}, \mathrm{H}-3\right), 6.19\left(\mathrm{~d}, 1 \mathrm{H}, J_{\mathrm{NH}-3^{\prime \prime}}=7.4 \mathrm{~Hz}, \mathrm{~N}-\mathrm{H}\right), 5.52\left(\mathrm{~d}, 1 \mathrm{H}, J_{1^{\prime}, 2^{\prime} \mathrm{ax}}=3.4 \mathrm{~Hz}, \mathrm{H}-1^{\prime}\right), 5.29$ (br s, $1 \mathrm{H}$, H-7), $5.04\left(\mathrm{br} \mathrm{s}, 1 \mathrm{H}, \mathrm{H}-4^{\prime \prime}\right), 4.99\left(\mathrm{br} \mathrm{s}, 1 \mathrm{H}, \mathrm{H}-\mathrm{1}^{\prime \prime}\right), 4.74\left(\mathrm{t}, 1 \mathrm{H}, J_{3^{\prime}, 4^{\prime}}=9.1\right.$ and $\left.J_{4^{\prime}, 5^{\prime}}=9.4 \mathrm{~Hz}, \mathrm{H}-4^{\prime}\right), 4.52(\mathrm{~s}$, $1 \mathrm{H}, \mathrm{OH}-9), 4.44\left(\mathrm{~m}, 1 \mathrm{H}, \mathrm{H}-3^{\prime \prime}\right), 4.08\left(\mathrm{~s}, 3 \mathrm{H}, \mathrm{OCH}_{3}\right), 4.04\left(\mathrm{q}, 1 \mathrm{H}, J_{5^{\prime \prime}, 6^{\prime \prime}}=6.5 \mathrm{~Hz}, \mathrm{H}-5^{\prime \prime}\right), 3.97(\mathrm{~m}, 1 \mathrm{H}$, $\left.J_{5^{\prime}, 6^{\prime}}=6.2 \mathrm{~Hz}, \mathrm{H}-5^{\prime}\right), 3.84\left(\mathrm{~m}, 1 \mathrm{H}, J_{3^{\prime}, 2^{\prime} \mathrm{ax}}=11.3\right.$ and $\left.J_{3^{\prime}, 2^{\prime} \mathrm{eq}}=5.1 \mathrm{~Hz}, \mathrm{H}-3^{\prime}\right), 3.25(\mathrm{~d}, 1 \mathrm{H}, \mathrm{H}-10 \mathrm{eq}), 2.98(\mathrm{~d}$, $\left.1 \mathrm{H}, J_{10 \mathrm{ax}, 10 \mathrm{eq}}=18.9 \mathrm{~Hz}, \mathrm{H}-10 \mathrm{ax}\right), 2.42\left(\mathrm{~s}, 3 \mathrm{H}, \mathrm{H}_{3}-14\right), 2.40 \sim 1.80\left(\mathrm{~m}, 6 \mathrm{H}, \mathrm{H}-8 \mathrm{eq}, \mathrm{H}-8 \mathrm{ax}, \mathrm{H}-2^{\prime}\right.$ eq, H-2'ax, $\mathrm{H}-2^{\prime \prime}$ eq and $\left.\mathrm{H}-2^{\prime \prime} \mathrm{ax}\right), 2.12$ (s, 3H, $\left.\mathrm{CH}_{3} \mathrm{CO}\right), 2.09$ (s, 3H, $\left.\mathrm{CH}_{3} \mathrm{CO}\right), 1.22\left(\mathrm{~d}, 3 \mathrm{H}, \mathrm{CH}_{3}{ }^{\prime}\right), 1.00\left(\mathrm{~d}, 3 \mathrm{H}, \mathrm{CH}_{3}{ }^{\prime \prime}\right)$; ${ }^{13} \mathrm{C} \mathrm{NMR}\left(\mathrm{Me}_{2} \mathrm{SO}-d_{6}\right): \delta 211.13(\mathrm{C}-13), 186.10(\mathrm{C}-5$ and $\mathrm{C}-12), 169.76\left(\mathrm{CH}_{3} \mathrm{CO}\right), 169.18\left(\mathrm{CH}_{3} \mathrm{CO}\right), 160.55$ (C-4), 156.18 and 154.61 (C-6 and C-11), 135.53, 134.77, 134.54 and 134.42 (C-2, C-6a, C-10a and C-12a), $120.00,118.94$ and 118.85 (C-1, C-4a and C-3), 110.45 and 110.39 (C-5a and C-11a), 99.85 and 97.57 (C-1' and $\left.\mathrm{C}-1^{\prime \prime}\right), 75.95,75.09,73.47,70.33,68.57,65.53$ and 64.24 (C-3', C-4', C-4", C-5", C-5', C-7 and C-9), $56.21\left(\mathrm{OCH}_{3}\right), 36.71$ and $35.93(\mathrm{C}-8$ and $\mathrm{C}-10), 31.59$ and $28.74\left(\mathrm{C}-2^{\prime}\right.$ and $\left.\mathrm{C}-2^{\prime \prime}\right), 23.80(\mathrm{C}-14), 20.42$ $\left(\mathrm{CH}_{3} \mathrm{CO}\right), 20.18\left(\mathrm{CH}_{3} \mathrm{CO}\right), 16.99$ and $16.16\left(\mathrm{C}-6^{\prime}\right.$ and $\left.\mathrm{C}-6^{\prime \prime}\right)$.

Anal Caled for $\mathrm{C}_{39} \mathrm{H}_{42} \mathrm{~F}_{3} \mathrm{NO}_{16}$ : C 55.91, H 5.05, N 1.67 .

Found:

C 55.78, H 5.08, N 1.65 .

7-O-[3-O-(3-Amino-2,3,6-trideoxy- $\alpha$-L-lyxo-hexopyranosyl)-2,6-dideoxy- $\alpha$-L-arabino-hexopyranosyl]daunomycinone Hydrochloride (15)

To a solution of $14(0.175 \mathrm{~g}, 0.21 \mathrm{mmol})$ in dry $\mathrm{MeOH}(12 \mathrm{ml})$, was added $0.5 \mathrm{M}$ methanolic $\mathrm{NaOMe}$ $(1 \mathrm{ml})$. The solution turned from red to purple. The reaction was monitored by TLC ( $3: 1$ toluene - acetone) and after 4 hours the reaction was terminated by adding Dry Ice whereupon the color changed from purple back to red. The solution was diluted with $\mathrm{CH}_{2} \mathrm{Cl}_{2}$ washed with water, dried $\left(\mathrm{Na}_{2} \mathrm{SO}_{4}\right)$, and evaporated to afford a red solid that was used in the next step without any further purification. The crude solid was suspended in $15 \mathrm{ml}$ of $0.1 \mathrm{~N}$ aqueous $\mathrm{NaOH}$. The color of the solution changed to purple. The reaction was monitored by TLC ( $3: 1$ toluene-acetone) until no more starting material could be detected ( 20 minutes). The reaction was stopped by adding $5 \% \mathrm{HCl}$ until the solution became red $(1.5 \mathrm{ml})$. The solution was then diluted with water $(20 \mathrm{ml})$ and washed with $\mathrm{CH}_{2} \mathrm{Cl}_{2}(3 \times 40 \mathrm{ml})$ and then extracted with 1-butanol until the aqueous solution became colorless. The 1-butanol solution was evaporated and the solid remaining was crystallized from $0.1 \mathrm{M} \mathrm{HCl}$ in $\mathrm{MeOH}$ and ether to give pure $15(45 \mathrm{mg}, 32 \%)$; mp $160 \sim 162^{\circ} \mathrm{C}$ (with decomposition); $[\alpha]_{\mathrm{D}}^{20}+368^{\circ}(c 0.05, \mathrm{MeOH}) ;{ }^{1} \mathrm{H}$ NMR $\left(\mathrm{CD}_{3} \mathrm{OD}\right): \delta 7.93(\mathrm{~d}, 1 \mathrm{H}$, $\left.J_{1,2}=6.8 \mathrm{~Hz}, \mathrm{H}-1\right), 7.82(\mathrm{dd}, 1 \mathrm{H}, \mathrm{H}-2), 7.54\left(\mathrm{~d}, 1 \mathrm{H}, J_{2,3}=8.1 \mathrm{~Hz}, \mathrm{H}-3\right), 5.38\left(\mathrm{~d}, 1 \mathrm{H}, J_{1^{\prime}, 2^{\prime} \mathrm{ax}}=3.3 \mathrm{~Hz}, \mathrm{H}-1^{\prime}\right)$, 5.16 (br s, $\left.1 \mathrm{H}, \mathrm{H}-1^{\prime \prime}\right), 5.10$ (br s, $\left.1 \mathrm{H}, \mathrm{H}-7\right), 4.02\left(\mathrm{~s}, 3 \mathrm{H}, \mathrm{OCH}_{3}\right), 3.96\left(\mathrm{~m}, 1 \mathrm{H}, J_{4^{\prime}, 5^{\prime}}=8.5\right.$ and $J_{5^{\prime}, 6^{\prime}}=6.2 \mathrm{~Hz}$, $\left.\mathrm{H}-5^{\prime}\right), 3.92\left(\mathrm{q}, 1 \mathrm{H}, J_{5^{\prime \prime}, 6^{\prime \prime}}=6.7 \mathrm{~Hz}, \mathrm{H}-5^{\prime \prime}\right), 3.78\left(\mathrm{~m}, 1 \mathrm{H}, J_{3^{\prime}, 2^{\prime} \mathrm{ax}}=11.7\right.$ and $\left.J_{3^{\prime}, 2^{\prime} \mathrm{eq}}=5.1 \mathrm{~Hz}, \mathrm{H}-3^{\prime}\right), 3.56(\mathrm{~m}$, $2 \mathrm{H}, \mathrm{H}-3^{\prime \prime}$ and $\left.\mathrm{H}-4^{\prime \prime}\right), 3.09\left(\mathrm{t}, 1 \mathrm{H}, J_{3^{\prime}, 4^{\prime}}=9.1 \mathrm{~Hz}, \mathrm{H}-4^{\prime}\right), 3.04(\mathrm{~d}, 1 \mathrm{H}, \mathrm{H}-10 \mathrm{eq}), 2.99\left(\mathrm{~d}, 1 \mathrm{H}, J_{10 \mathrm{ax}, 10 \mathrm{eq}}=18.0 \mathrm{~Hz}\right.$, $\mathrm{H}-10 \mathrm{ax}), 2.35\left(\mathrm{~s}, 3 \mathrm{H}, \mathrm{H}_{3}-14\right), 2.32\left(\mathrm{~d}, 1 \mathrm{H}, J_{8 \mathrm{eq}, 8 \mathrm{ax}}=13.0 \mathrm{~Hz}, \mathrm{H}-8 \mathrm{eq}\right), 2.22\left(\mathrm{dd}, 1 \mathrm{H}, J_{8 \mathrm{ax}, 7}=4.7 \mathrm{~Hz}, \mathrm{H}-8 \mathrm{ax}\right)$, $2.16\left(\mathrm{dd}, 1 \mathrm{H}, J_{2^{\prime} \text { eq, } 2^{\prime} \text { ax }}=14.0 \mathrm{~Hz}, \mathrm{H}-2^{\prime} \mathrm{eq}\right), 1.90\left(\mathrm{~m}, 2 \mathrm{H}, \mathrm{H}-2^{\prime \prime}\right.$ eq and $\left.\mathrm{H}-2^{\prime \prime} \mathrm{ax}\right), 1.72\left(\mathrm{~m}, 1 \mathrm{H}, \mathrm{H}-2^{\prime} \mathrm{ax}\right), 1.30$ (d, 3H, $\left.\mathrm{CH}_{3}{ }^{\prime}\right), 1.06\left(\mathrm{~d}, 3 \mathrm{H}, \mathrm{CH}_{3}{ }^{\prime \prime}\right) ;{ }^{13} \mathrm{C}$ NMR $\left(\mathrm{CD}_{3} \mathrm{OD}\right): \delta 213.58(\mathrm{C}-13), 187.87$ and $187.54(\mathrm{C}-5$ and C-12), 162.36 (C-4), 157.36 and 156.14 (C-6 and C-11), 137.14, 136.20, 135.80 and 135.73 (C-2, C-6a, C-10a and C-12a), 121.39, 120.48 and 120.20 (C-1, C-4a and C-3), 112.32 and 112.08 (C-5a and C-11a), 102.20 and $99.02\left(\mathrm{C}-1^{\prime}\right.$ and $\left.\mathrm{C}-1^{\prime \prime}\right), 77.90,77.56,76.96,71.63,70.31,67.83$ and $67.27\left(\mathrm{C}-3^{\prime}, \mathrm{C}-4^{\prime}, \mathrm{C}-4^{\prime \prime}, \mathrm{C}-5^{\prime \prime}\right.$, $\mathrm{C}-5^{\prime}, \mathrm{C}-7$ and $\left.\mathrm{C}-9\right), 57.09\left(\mathrm{OCH}_{3}\right), 38.24$ and $36.97(\mathrm{C}-8$ and $\mathrm{C}-10), 33.49$ and $29.42\left(\mathrm{C}-2^{\prime}\right.$ and $\left.\mathrm{C}-2^{\prime \prime}\right), 24.60$ (C-14), 18.10 and 16.89 (C-6' and C-6").

Anal Calcd for $\mathrm{C}_{33} \mathrm{H}_{39} \mathrm{NO}_{13}$ : 657.2517 , high resolution $\mathrm{FAB}$ mass spectra: 657.2500 .

Acknowledgment

The authors thank Dr. DANIEL LEDNICER of the National Cancer Institute for arranging the biological testing. 


\section{References}

1) El Khadem, H. S. (Ed): Anthracycline Antibiotics. Academic Press, 1982

2) Arcamone, F.; G. Cassinelit, S. Penco \& L. Tognoli (Societa Farmaceutici Italia S.p.A.): Antibiotic from Streptomyces fermentations. Ger. Offen. 1923885 , Jan. 29, 1970

3) Penco, S.; G. Franchi \& F. ARcamone (Societa Farmaceutici Italia S.p.A.): Anthracycline disaccharide derivatives. Ger. Offen. 2751 395, May 18, 1978

4) Fuchs, E. F.; D. Horton, W. WeCkerle \& E. Winter-Mihaly: Synthesis and antitumor activity of sugar-ring hydroxyl analogues of daunorubicin. J. Med. Chem. 22: 406 411, 1979

5) Horton, D.; W. Priebe \& O. Varela: 3'-Deamino-4'-epi-3'-hydroxy-daunorubicin and -doxorubicin. Synthesis and antitumor activity. J. Antibiotics 37: 1635 1641, 1984

6) Horton, D.; W. Priebe \& M. Sznaidman: A new approach to 2-deoxyglycosides permitting access to anthracycline glycosides specifically labeled at the $2^{\prime}$-position. Carbohydr. Res. 187: 149 153, 1989

7) Thiem, J.; H. Karl \& J. Schwentner: Synthese $\alpha$-verknüpfter $2^{\prime}$-deoxy-2'-iododisaccharide. Synthesis 1978 : $696 \sim 698,1978$

8) ThIEM, J. \& M. GERKEn: Synthesis of the E-D-C trisaccharide unit of aureolic acid cytostatics. J. Org. Chem. 50: $954 \sim 958,1985$

9) Thiem, J. \& W. KLAFFKE: Facile stereospecific synthesis of deoxyfucosyl disaccharide units of anthracyclines. J. Org. Chem. 54: 2006 2009, 1989

10) Horton, D. \& W. Priebe: Oxyhalogenation of glycals for the synthesis of antitumor-active 2 '-halo daunorubicin analogs. Carbohydr. Res. 136: 391 396, 1985

11) Berkowitz, D. B.; S. J. DanishefSKy \& G. K. Schulte: A route to artificial glycoconjugates and oligosaccharides via enzymatically resolved glycals: dramatic effects of the handedness of the sugar domain upon the properties of an anthracycline drug. J. Am. Chem. Soc. 114: 4518 4529, 1992

12) Tatsuta, K.; K. Fujimoto, M. Kinoshita \& S. UmeZawa: A novel synthesis of 2-deoxy- $\alpha$-glycosides. Carbohydr. Res. 54: $85 \sim 104,1977$

13) Horton, D.; W. Priebe \& M. Sznaidman: Steric and conformational effects in the dehalogenation of 2-halo sugar derivatives with tributylstannane. J. Org. Chem., 58: 1821 1826, 1993

14) Horton, D.; W. Priere \& M. Sznaidman: Preparation of 4-O-acetyl-1,5-anhydro-2,3,6-trideoxy-3trifluoroacetamido-L-lyxo-hex-1-enitol, a key intermediate in synthesis of daunosamine glycosides. Carbohydr. Res. 187: 145 148, 1989

15) Horton, D.; W. Priebe \& O. Varela: Selective acylation of 6-deoxyglycals. Carbohydr. Res. 144: 317 324, 1985

16) Horton, D.; W. Priebe \& M. Sznaidman: Iodoalkoxylation of 1,5-anhydro-2-deoxy-hex-1-enitols (glycals). Carbohydr. Res. 205: $71 \sim 86,1990$

17) Horton, D.; W. Priebe \& O. Varela: Halogenation of 1,5-anhydroex-1-enitols (glycals). Influence of the C-6 substituent. J. Org. Chem. 51: 3479 3485, 1986

18) Borvin, J.; C. Monneret \& M. PAïs: Approche de la synthese d'anthracyclines oligosaccharidiques. Hemisynthese de la 4'-O-(2-desoxy-L-fucosyl) daunorubicine. Tetrahedron 1981: 4219 4228, 1981

19) BERGE, J. M. \& S. M. ROBERTS: Recommended work-up procedure for reductions employing tri- $n$-butyltin hydride. Synthesis 1979: $471 \sim 472,1979$ 